\title{
Study of large geomagnetic storms (GMSs) and their association with different solar source activities observed during solar cycle
}

\author{
L.K. Borker ${ }^{1,}$ S.K. Khandayat ${ }^{2,}$ and S.C. Dubey ${ }^{3}$, \\ ${ }^{1}$ Department of Physics, Govt. College Umaria (M.P.) - 484661 India. \\ ${ }^{2}$ Department of Physics, Govt. College Nainpur, Mandla (M.P.) \\ ${ }^{3}$ Department of Physics, SGS Govt. PG College Sidhi (M.P.)-486661, India
}

\begin{abstract}
Geomagnetic storms (GMSs) are stimulated by higher solar wind flow speed $\left(\boldsymbol{V}_{S W}\right)$ and by a southward-directed interplanetary magnetic field $\left(\mathbf{I M F} \boldsymbol{B}_{z}\right)$. Two kinds of flows dominate the large scale structure of solar wind: corotating flows and transient disturbances. Corotating flows are associated with spatial variability in coronal expansion and solar rotation, whereas transient disturbances are associated with episodic ejections of material into interplanetary space from coronal regions. The high speed streams originated from coronal interaction regions (CIRs) and transient disturbances are mostly caused by fast coronal mass ejections (CMEs). CMEs are the crucial link between solar activity and transient interplanetary disturbances and are responsible for major GMSs. CMEs eject magnetic flux into interplanetary space that produces abrupt increase in the northward component of Earth's magnetic field. There are two types of geomagnetic field variations termed as long-time variation and storm-time variations. The long-term variations are very useful to solar cyclical study of geomagnetic field variation as well as change in polarity of the Sun, climate change, plants growth rate and geological change of Earth's pole. The storm time variations also known as geomagnetic storm deals the various characteristics of GMSs and their connection with solar source activities and interplanetary magnetic fields. These variations are directly affect us and shows adverse effect in satellites, communication system and power losses. In the present study, we have analysed large geomagnetic storms $(G M S s)$ associated with storm time index $\left(D_{s t}\right)$ decrease of more than $100 \mathrm{nT}$ observed during solar cycle 23 and found 91 geomagnetic storms falling in our selection criteria. We have analyzed different characteristics of above GMSs and their association with different solar source activities.
\end{abstract}

Keywords: CMEs, CIRs, $D_{s t}, I M F$, GMSs.

\section{Introduction}

The geomagnetosphere and upper atmosphere can be greatly perturbed by variations in the solar wind caused by disturbances on the Sun. Changes in the orientation of the interplanetary magnetic field (IMF) and major increases in the velocity and density of solar wind particles striking the magnetosphere result in geomagnetic storms (GMSs). GMSs are seen at the surface of the Earth as perturbations in the components of the geomagnetic field, caused by electric currents flowing in the magnetosphere and upper atmosphere. Major GMSs are the most dramatic manifestation of solar terrestrial coupling. They involve the injection of large amounts of energy from the solar wind into the Earth's magnetosphere, ionosphere and thermosphere. Intense GMSs also have major effects on technical systems in space. An important challenge to solar-terrestrial physicist is to understand which solar and interplanetary process caused the geomagnetic activity. There are many solar source activities which are defined as cause of large geomagnetic disturbances as discussed by many authors. The transfer energy and plasma from Sun to Earth is also interesting. Throughout the heliosphere, the solar wind plasma carries embedded within it solar magnetic field lines. The transfer of energy, momentum and mass from the Sun to Earth for a number of solar perturbations under a variety of interplanetary configurations is also a major scientific objective.

Solar output in term of solar plasma and magnetic field ejected out into interplanetary medium consequently create the perturbation in the geomagnetic field. In recent years, these in situ data have resulted in explosive growth in our knowledge and understanding of solar-interplanetary-terrestrial process. There is a variety of development of GMSs and many authors have suggested several solar and interplanetary causes. The magnetic reconnection provides opportunity to enter solar plasma within geomagnetosphere. Considering various latest theories and mechanism, we have analysed large geomagnetic storms associated with $\mathrm{D}_{\mathrm{st}}$ decrease of more than $100 \mathrm{nT}$, observed during solar cycle 23 and its various associations. 


\section{Selection Criteria And Data Sources}

In the present analysis, we have sorted out GMSs associated with $\mathrm{D}_{\mathrm{st}}$ decrease of more than $100 \mathrm{nT}$, IMF B $\geq 10 \mathrm{nT}$ with time duration greater than 3 hours, during the period 1996-2007. Different types of solar and geomagnetic indices data are available from different solar and geomagnetic observatories word-wide. The International Service of Geomagnetic Indices (ISGI) and ISGI collaborating institutes have continuously published these indices in the form of data series. Some data is directly available on the internet and some is available on request to principal investigator. During the aforesaid period, we find 91 are large GMSs $\left(D_{s t} \leq-100\right.$ nT). A list of all those selected GMSs and their associative properties are summarized in Table 1. In the table, Column (1) and (2) presents serial number and observed date of GMSs. Column (3) presents magnitude of GMSs ( $\mathrm{D}_{\mathrm{st}}$ in net). Column (4) presents the associated solar driver of large GMSs. Peak velocity of CME is noted in column (5). Solar flare class, source region and their co-ordinates are given in column (6-8) respectively.

Table 1: The list of 91 large GMSs and their characteristics, observed during solar cycle 23.

\begin{tabular}{|c|c|c|c|c|c|c|c|}
\hline $\begin{array}{l}\text { Storm } \\
\text { No. }\end{array}$ & $\begin{array}{l}\text { Date of } \quad \mathbf{D}_{\text {st }} \\
\text { storm }\end{array}$ & $\begin{array}{l}\text { Magnitude } \\
\text { (nT) }\end{array}$ & $\begin{array}{l}\text { Solar } \\
\text { Driver }\end{array}$ & $\begin{array}{l}\text { CME } \\
\text { Velocity } \\
\text { Km/sec. }\end{array}$ & Flare Class & $\begin{array}{l}\text { Source } \\
\text { Region }\end{array}$ & $\begin{array}{l}\text { Source } \\
\text { Coordinate }\end{array}$ \\
\hline (1) & (2) & (3) & (4) & (5) & (6) & (7) & (8) \\
\hline 01 & $23 / 10 / 96$ & -105 & CIR & - & - & $\mathrm{CH}$ & Unknown \\
\hline 02 & $21 / 04 / 97$ & -120 & CME-S & 87 & No & Unknown & Unknown \\
\hline $\mathbf{0 3}$ & $15 / 05 / 97$ & -115 & CME-S & 464 & C 1.3 & AR8038 & N21W08 \\
\hline 04 & $11 / 10 / 97$ & -130 & CME-S & 293 & No & $\mathrm{QS}$ & S27W05 \\
\hline 05 & $07 / 11 / 97$ & -110 & CME-S & 785 & X 2.1 & AR8100 & S14W33 \\
\hline 06 & $23 / 11 / 97$ & -108 & CME-S & DG & C 1.6 & AR8108 & N20E05 \\
\hline 07 & $18 / 02 / 98$ & -100 & CME-S & 63 & No & Unknown & Unknown \\
\hline 08 & $10 / 03 / 98$ & -116 & CIR & - & No & $\mathrm{CH}$ & S30 \\
\hline \multirow[t]{4}{*}{09} & \multirow{4}{*}{$04 / 05 / 98$} & \multirow[t]{4}{*}{-205} & \multirow[t]{4}{*}{ CME-M } & 938 & X 1.1 & AR8210 & S15W15 \\
\hline & & & & 542 & C 5.4 & AR8210 & S19W09 \\
\hline & & & & 585 & M 1.2 & AR8210 & S18W04 \\
\hline & & & & 1374 & M 6.8 & AR8210 & S18E20 \\
\hline \multirow[t]{2}{*}{10} & \multirow[t]{2}{*}{$26 / 06 / 98$} & \multirow[t]{2}{*}{-101} & \multirow[t]{2}{*}{ CME-M } & 289 & No & QS & S58W05 \\
\hline & & & & 192 & No & AR8243 & N15W30 \\
\hline 11 & $06 / 08 / 98$ & -138 & CME-M & DG & No & DG & DG \\
\hline 12 & $07 / 08 / 98$ & -108 & CIR & - & No & $\mathrm{CH}$ & DG \\
\hline 13 & $27 / 08 / 98$ & -155 & CME-S & - & X 1.0 & AR8307 & N35E09 \\
\hline 14 & $25 / 09 / 98$ & -207 & CME-S & - & M 7.1 & AR8340 & N18E09 \\
\hline 15 & 19/10/98 & -112 & CME-S & 362 & No & $\mathrm{QS}$ & N10E10 \\
\hline \multirow[t]{2}{*}{16} & \multirow[t]{2}{*}{ 08/11/98 } & \multirow[t]{2}{*}{-149} & \multirow[t]{2}{*}{ CME-M } & 523 & C 5.2 & AR8375 & N17E01 \\
\hline & & & & 1119 & M 8.4 & AR8375 & $\mathrm{N} 22 \mathrm{~W} 18$ \\
\hline 17 & $09 / 11 / 98$ & -142 & CME-S & 1119 & M 8.4 & AR8375 & N22W18 \\
\hline 18 & $13 / 11 / 98$ & -131 & CME-S & 325 & No & QS & N18E00 \\
\hline 19 & $13 / 01 / 99$ & -112 & CME-S & - & No & DG & DG \\
\hline 20 & $18 / 02 / 99$ & -123 & CME-S & - & M 3.2 & AR8458 & S23W14 \\
\hline 21 & $22 / 09 / 99$ & -173 & CME-S & 604 & C 2.8 & $\mathrm{QS}$ & S21W05 \\
\hline 22 & $22 / 10 / 99$ & -237 & CME-S & 144 & No & $\mathrm{QS}$ & S26E08 \\
\hline 23 & $13 / 11 / 99$ & -106 & CME-M & - & - & DG & DG \\
\hline 24 & $12 / 02 / 2 \mathrm{~K}$ & -133 & CME-S & 944 & C 7.3 & AR8858 & N22E03 \\
\hline 25 & $07 / 04 / 2 \mathrm{~K}$ & -288 & CME-S & 1188 & C 9.7 & AR8933 & N16W66 \\
\hline \multirow[t]{2}{*}{26} & \multirow[t]{2}{*}{$24 / 05 / 2 \mathrm{~K}$} & \multirow[t]{2}{*}{-147} & \multirow[t]{2}{*}{ CME-M } & 557 & C 7.6 & AR8998 & S15W08 \\
\hline & & & & 649 & C 6.3 & AR9004 & N20W22 \\
\hline 27 & $16 / 07 / 2 \mathrm{~K}$ & -301 & CME-S & 1674 & X 5.7 & AR9077 & N22W07 \\
\hline 28 & $11 / 08 / 2 \mathrm{~K}$ & -106 & CME-S & 281 & C 1.4 & Unknown & Unknown \\
\hline 29 & $12 / 08 / 2 \mathrm{~K}$ & -235 & CME-S & 702 & C 2.3 & AR9114 & N11W11 \\
\hline \multirow[t]{4}{*}{30} & \multirow[t]{4}{*}{$17 / 09 / 2 \mathrm{~K}$} & \multirow[t]{4}{*}{-201} & \multirow[t]{4}{*}{ CME-M } & 1215 & M 5.9 & AR9165 & N14W07 \\
\hline & & & & 285 & C 7.4 & AR9165 & N12E04 \\
\hline & & & & 481 & M 2.0 & AR9165 & N12E07 \\
\hline & & & & 633 & C 9.5 & AR9165 & N13E08 \\
\hline
\end{tabular}


Study of large geomagnetic storms (GMSs) and their association with different solar source

\begin{tabular}{|c|c|c|c|c|c|c|c|}
\hline \multirow[t]{2}{*}{31} & \multirow[t]{2}{*}{$05 / 10 / 2 \mathrm{~K}$} & \multirow[t]{2}{*}{-182} & \multirow[t]{2}{*}{ CME-M } & 173 & No & Unknown & Unknown \\
\hline & & & & 586 & C 5.0 & $\mathrm{QS}$ & S27E33 \\
\hline 32 & $14 / 10 / 2 \mathrm{~K}$ & -107 & CME-S & 798 & C 6.7 & AR9182 & N01W14 \\
\hline 33 & $29 / 10 / 2 \mathrm{~K}$ & -127 & CME-S & 770 & C 4.0 & QS & N06W60 \\
\hline 34 & $06 / 11 / 2 \mathrm{~K}$ & -159 & CME-S & 291 & No & Unknown & Unknown \\
\hline \multirow[t]{4}{*}{35} & \multirow{4}{*}{$29 / 11 / 2 \mathrm{~K}$} & \multirow[t]{4}{*}{-130} & \multirow{4}{*}{ CME-M } & 675 & M 3.5 & AR9236 & N18W24 \\
\hline & & & & 671 & X 1.9 & AR9236 & N20W23 \\
\hline & & & & 495 & No & QS & S30W40 \\
\hline & & & & 980 & $\mathrm{X} 4.0$ & AR9236 & N18W38 \\
\hline 36 & $20 / 03 / 01$ & -149 & CME-S & 271 & No & Unknown & Unknown \\
\hline \multirow[t]{2}{*}{37} & \multirow[t]{2}{*}{$31 / 03 / 01$} & \multirow[t]{2}{*}{-387} & \multirow{2}{*}{ CME-M } & 942 & X 1.7 & AR9393 & N20W19 \\
\hline & & & & 519 & M 4.3 & AR9393 & N16E03 \\
\hline \multirow[t]{2}{*}{38} & \multirow[t]{2}{*}{$11 / 04 / 01$} & \multirow[t]{2}{*}{-271} & \multirow[t]{2}{*}{ CME-M } & 2411 & X 2.3 & AR9415 & S23W09 \\
\hline & & & & 1192 & M 7.9 & AR9415 & S21W04 \\
\hline 39 & $18 / 04 / 01$ & -114 & CME-S & 1199 & X 14.4 & AR9415 & S20W85 \\
\hline 40 & $22 / 04 / 01$ & -102 & CME-S & - & No & Unknown & Unknown \\
\hline 41 & $17 / 08 / 01$ & -105 & CME-S & 618 & C 2.3 & AR9577 & N16W36 \\
\hline 42 & $26 / 09 / 01$ & -102 & CME-S & 2402 & X 2.6 & AR9632 & S16E23 \\
\hline 43 & $01 / 10 / 01$ & -148 & CME-S & 846 & M 3.3 & AR9636 & N08E19 \\
\hline 44 & $03 / 10 / 01$ & -166 & CME-S & 509 & M 1.8 & AR9636 & N13E03 \\
\hline 45 & $21 / 10 / 01$ & -187 & CME-S & 901 & X 1.6 & AR9661 & N15W29 \\
\hline \multirow[t]{2}{*}{46} & $28 / 10 / 01$ & -157 & CME-M & 1092 & X 1.3 & AR9672 & S18W19 \\
\hline & & & & 597 & C 2.6 & AR9675 & S13E27 \\
\hline 47 & $06 / 11 / 01$ & -292 & CME-M & 1810 & X 1.0 & AR9684 & N06W18 \\
\hline & & & & 457 & No & DG & DG \\
\hline 48 & $24 / 11 / 01$ & -221 & CME-M & 1437 & M 9.9 & AR9704 & S14W36 \\
\hline & & & & 1443 & M 3.8 & AR9698 & S25W67 \\
\hline 49 & $24 / 03 / 02$ & -101 & CME-M & 860 & M 1.0 & AR9866 & S10W58 \\
\hline & & & & 603 & No & AR9871 & S21W15 \\
\hline 50 & $18 / 04 / 02$ & -126 & CME-S & 720 & M 1.2 & AR9906 & S15W01 \\
\hline 51 & $20 / 04 / 02$ & -151 & CME-S & 1240 & M 2.6 & AR9906 & S14W34 \\
\hline 52 & $11 / 05 / 02$ & -102 & CME-S & 614 & C 4.2 & AR9934 & S12W07 \\
\hline 53 & $23 / 05 / 02$ & -108 & CME-M & 1246 & C 9.7 & AR9948 & S25W64 \\
\hline & & & & 1557 & C 5.0 & QS & S22W53 \\
\hline 54 & $02 / 08 / 02$ & -102 & CME-M & 562 & No & QS & N34W36 \\
\hline & & & & 360 & No & Unknown & Unknown \\
\hline 55 & $21 / 08 / 02$ & -106 & CME-S & 1585 & M 5.2 & AR0069 & S14E20 \\
\hline 56 & $04 / 09 / 02$ & -104 & CIR & - & - & $\mathrm{CH}$ & S15 \\
\hline 57 & $08 / 09 / 02$ & -170 & CME-M & 1748 & C 5.2 & AR0102 & N09E28 \\
\hline & & & & - & - & - & - \\
\hline 58 & $01 / 10 / 02$ & -183 & CME-S & 178 & No & Unknown & Unknown \\
\hline 59 & $04 / 10 / 02$ & -143 & CME-S & 258 & No & QS & S17W17 \\
\hline 60 & $07 / 10 / 02$ & -107 & CIR & - & - & $\mathrm{CH}$ & S07 \\
\hline 61 & $14 / 10 / 02$ & -102 & CIR & - & - & $\mathrm{CH}$ & S26 \\
\hline 62 & $21 / 11 / 02$ & -126 & CIR & - & - & $\mathrm{CH}$ & S04 \\
\hline 63 & $30 / 05 / 03$ & -131 & CME-M & 1366 & X 3.6 & AR0365 & S11W12 \\
\hline & & & & 964 & X 1.3 & AR0365 & S00W17 \\
\hline & & & & 509 & M 1.6 & AR0365 & S07W14 \\
\hline 64 & $18 / 06 / 03$ & -145 & CME-M & 875 & No & QS & N22W15 \\
\hline & & & & 2053 & X 1.3 & AR0386 & S07E80 \\
\hline 65 & $12 / 07 / 03$ & -118 & CIR & - & - & $\mathrm{CH}$ & N04 \\
\hline 66 & $16 / 07 / 03$ & -117 & CIR & - & - & $\mathrm{CH}$ & N03 \\
\hline 67 & $18 / 08 / 03$ & -168 & CME-S & 378 & No & Unknown & Unknown \\
\hline 68 & $29 / 10 / 03$ & -363 & CME-S & 2459 & X 17.2 & AR0486 & S16E08 \\
\hline 69 & $30 / 10 / 03$ & -401 & CME-S & 2029 & X 10.0 & AR0486 & S15W02 \\
\hline 70 & $20 / 11 / 03$ & -472 & CME-S & 1660 & M 3.9 & AR0501 & N00E18 \\
\hline
\end{tabular}


Study of large geomagnetic storms (GMSs) and their association with different solar source

\begin{tabular}{|c|c|c|c|c|c|c|c|}
\hline 71 & $22 / 01 / 04$ & -149 & CME-S & 965 & C 5.5 & AR0540 & S13W11 \\
\hline 72 & $11 / 02 / 04$ & -109 & CIR & - & - & $\mathrm{CH}$ & N02 \\
\hline 73 & $04 / 04 / 04$ & -112 & CME-S & - & C 3.4 & AR0582 & N16W10 \\
\hline 74 & $23 / 07 / 04$ & -101 & CME-S & 710 & M 8.6 & AR0652 & N10E35 \\
\hline 75 & $25 / 07 / 04$ & -148 & CME-S & 899 & C 5.3 & AR0652 & N02E08 \\
\hline 76 & $27 / 07 / 04$ & -181 & CME-S & 1333 & M 1.1 & AR0652 & N04W30 \\
\hline 77 & $30 / 08 / 04$ & -126 & CME-S & 108 & No & Unknown & Unknown \\
\hline \multirow[t]{2}{*}{78} & \multirow[t]{2}{*}{ 08/11/04 } & \multirow[t]{2}{*}{-373} & \multirow{2}{*}{ CME-M } & 1055 & M 5.4 & AR0696 & N08E18 \\
\hline & & & & 653 & C 6.3 & AR0696 & S09E28 \\
\hline \multirow[t]{2}{*}{79} & \multirow[t]{2}{*}{$10 / 11 / 04$} & \multirow[t]{2}{*}{-289} & \multirow{2}{*}{ CME-M } & 1759 & X 2.0 & AR0696 & N09W17 \\
\hline & & & & 1111 & M 9.3 & AR0696 & N09E05 \\
\hline \multirow[t]{2}{*}{80} & \multirow[t]{2}{*}{$18 / 01 / 05$} & \multirow[t]{2}{*}{-121} & \multirow{2}{*}{ CME-M } & 2861 & X 2.6 & AR0720 & N16W05 \\
\hline & & & & 2049 & M 8.6 & AR0720 & N16E04 \\
\hline 81 & $22 / 01 / 05$ & -105 & CME-S & 882 & X 7.1 & AR0720 & N12W58 \\
\hline 82 & $08 / 05 / 05$ & -127 & CIR & - & - & $\mathrm{CH}$ & N10 \\
\hline 83 & $15 / 05 / 05$ & -263 & CME-S & 1128 & M 8.0 & AR0759 & N12E12 \\
\hline 84 & $20 / 05 / 05$ & -103 & CME-S & 405 & C 1.2 & AR0759 & N13W29 \\
\hline 85 & $30 / 05 / 05$ & -138 & CME-S & 586 & B 7.5 & AR0767 & S13E13 \\
\hline 86 & $13 / 06 / 05$ & -106 & CME-S & 377 & C 1.4 & AR0767 & N07E12 \\
\hline \multirow[t]{2}{*}{87} & \multirow[t]{2}{*}{$24 / 08 / 05$} & \multirow[t]{2}{*}{-216} & \multirow[t]{2}{*}{ CME-M } & 1194 & M 2.6 & AR0798 & S11W54 \\
\hline & & & & 2378 & M 5.6 & AR0798 & S12W60 \\
\hline 88 & $31 / 08 / 05$ & -131 & CIR & - & - & $\mathrm{CH}$ & S12 \\
\hline 89 & $11 / 09 / 05$ & -147 & CME-S & 2257 & X 6.2 & AR0808 & S10E58 \\
\hline 90 & $14 / 04 / 06$ & -111 & CME-S & - & - & - & - \\
\hline 91 & $15 / 12 / 06$ & -146 & CME-S & - & - & - & - \\
\hline \multicolumn{4}{|c|}{$\begin{array}{l}\text { CME-S Single CME/ICME } \\
\text { CME-M Multiple CME/ICME } \\
\text { QS Quit Sun region }\end{array}$} & \multicolumn{4}{|c|}{$\begin{array}{l}\text { Class of Solar Flares } \sim \mathbf{B}, \mathbf{C}, \mathbf{X} \text { and } \mathbf{M} \text { respectively } \\
\mathbf{C I R} \sim \text { Coronal holes/coronal interaction region } \\
\text { DG/- Data Gap }\end{array}$} \\
\hline
\end{tabular}

III. Association Of Large GMSs During Different Phases Of Solar Cycle 23

On the basis of comprehensive study of above selected 91 large GMSs observed during solar cycle 23, following conclusions are drawn:

[1] Out of selected $\mathbf{9 1}$ large GMSs events, $\mathbf{5 7 \%}$ were sudden commencement type and rest $\mathbf{4 3} \%$ were gradual commencement type.

[2] The long-term behaviour of occurrence of sudden commencement and gradual commencement GMSs haven't significant correlation between the maximum and minimum phases for solar cycle 23.

[3] Maximum number of sudden commencement GMSs having their initial phase duration lies between 0-2 hr.

[4] The main phase duration for maximum number of large GMSs lies between 7-12 hr.

[5] The best recovery phase duration for maximum number of large GMSs lies between 73-96 hr (3-4) days.

[6] The main phase duration is always less than the recovery phase duration and the GMSs associated with SSC shows faster recovery in comparison to other GMSs that is not associated with SSC. The study of semi-annual variation of GMSs have important role in space weather prediction.

[7] The semi-annual variation of large GMSs shows nearly a cyclic variation peaking around April and October months. This result is in good agreement with the findings of Russell-McPherron (1973) and Crooker et al (1992).

[8] In the first half-annual part (January-June), the maximum number of GMSs occurs during equinoctial April and May. During the next half-annual part (July-December), occurrence rate for large GMSs events is higher during solstitial October and November. This result does not agree with the earlier results obtained by Crooker et al (1992).

[9] It is also found that the 56\% large GMSs occurring during equinoctial and solstitial months.

Association of yearly occurrence of large GMSs with yearly mean sunspot number observed during 1996-2007 is shown in Figure 1. Figure 2 shows the occurrence of large GMSs during different phases of solar cycle 22 and 23. During ascending and maximum phase of solar cycle 22, we observed 20 and 50 GMSs, whereas during its declining phase 29 GMSs were observed. During ascending and maximum phase of solar cycle 23, we have observed 23 and 12 GMSs, whereas during its declining phase 56 GMSs were observed. So, the solar cycle 23 is remarkable for occurrence of large GMSs during its declining phase and shows controversial result measured for past solar cycle 22. From the listed GMSs (Table 1) it is seen that out of 11 severe GMSs $\left(D_{\text {st }} \leq-250 \mathrm{nT}\right)$ observed during solar cycle 23, 9 were observed during its declining phase. The largest GMS of solar cycle 23 were also observed on its declining phase. This GMS was observed on $20^{\text {th }}$ November 2003 (20:00 UT) with a $\mathrm{D}_{\mathrm{st}}$ index of $-472 \mathrm{nT}, \mathrm{A}_{\mathrm{p}}$ index of 300 and $\mathrm{K}_{\mathrm{p}}$ index of 8.7. It was caused by CMEs from active region 0501 had a sky-plane speed of $\sim 1660 \mathrm{~km} \mathrm{~s}^{-1}$, but the associated magnetic cloud (MC) arrived with a speed of only $730 \mathrm{~km} \mathrm{~s}^{-1}$. The $\mathrm{MC}$ at $1 \mathrm{AU}$ had a high magnetic field $(\sim 56 \mathrm{nT})$ and high inclination to the ecliptic plane. 


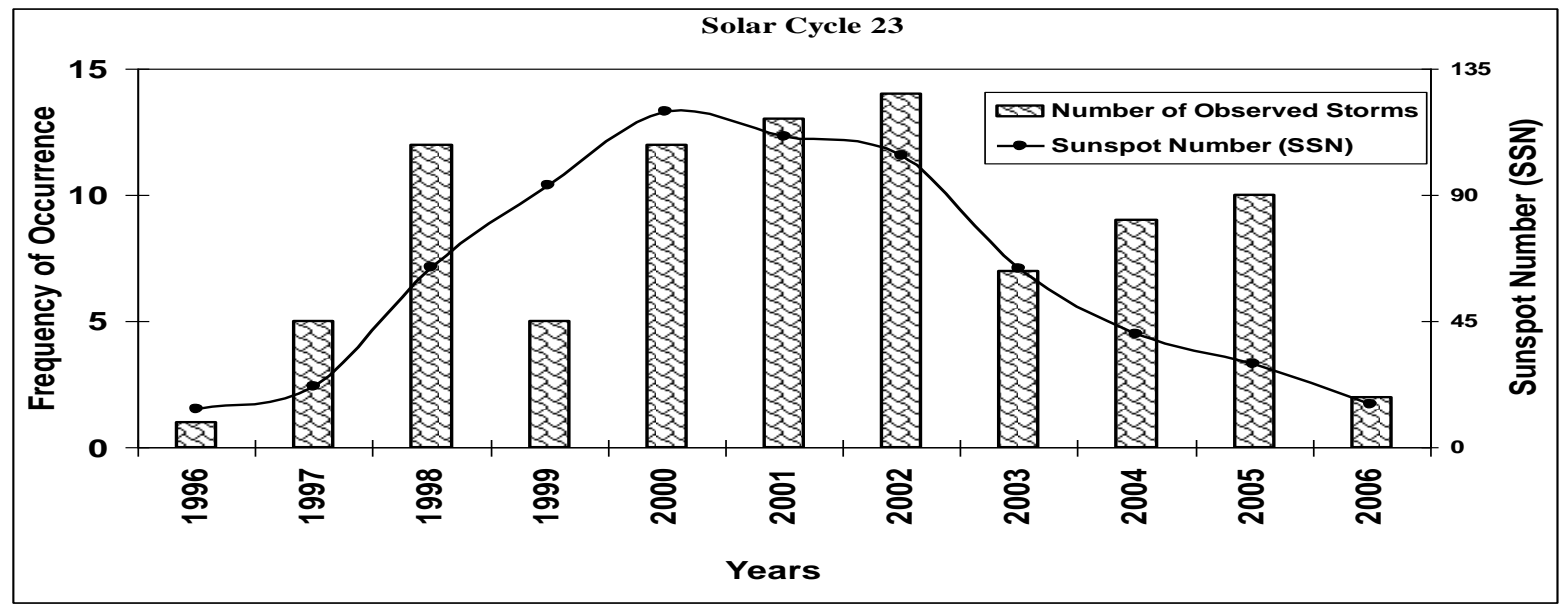

Figure 1: Shows the yearly occurrence of large GMSs and their association With 11-year sunspot cycle, observed during solar cycle 23 .

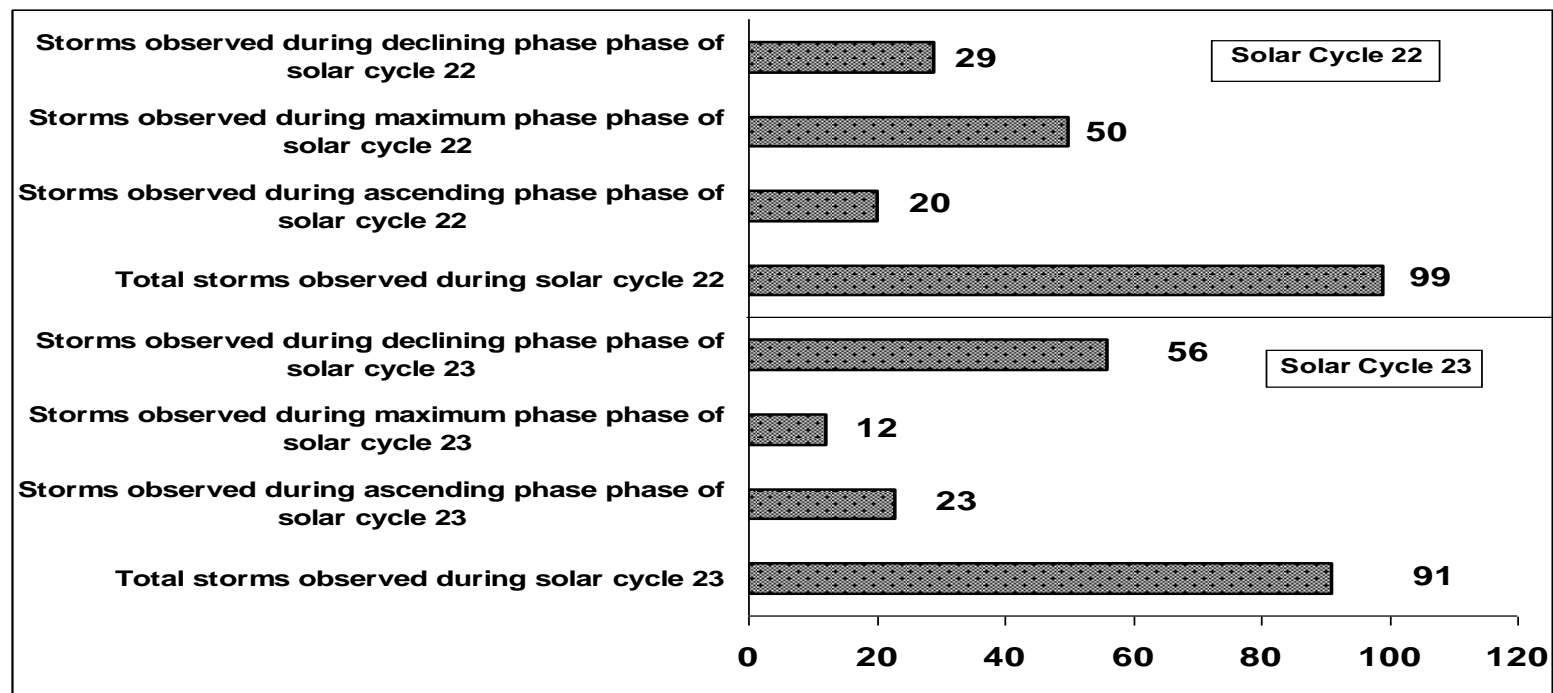

Figure 2: Shows the occurrence of large GMSs during different phases of solar cycle 22 and 23.

\section{Solar Proton Events (SPEs) and Their Impact On HF Communications}

Solar Proton Events (SPEs) occurs when high-energy protons are ejected from the Sun's surface during fast solar eruptions and causes geomagnetic and ionospheric disturbances on large scale. These effects are similar to auroral events, the difference being that electrons and not protons are involved. These events typically occur at the north pole, south pole, and South Atlantic magnetic anomaly, where the Earth's magnetic field is lowest. The more severe SPEs events can cause widespread disruption to electrical grids and the propagation of electromagnetic signals. The SPE is the energetic outbursts as a result of acceleration and heating of solar plasma during solar flares and CMEs. The first observation of SPE was recorded by Forbush (1946) in the form of abrupt enhancement in the intensity in ground-level ion chambers during large solar flares that occurred in February and March 1942. The SEPs are classified as the gradual and impulsive. SPEs associated with solar flares are called impulsive where as those associated with CMEs are known as gradual (Cliver, 1983, Cane et al., 1986 and Kahler, 1992).

In the present work, we have studied the general characteristics of SPEs occurred during past solar cycle 23. Occurrence of SPEs are directly associated with fast solar eruptions. Occurrence of fast solar eruptions varies with 11-year sunspot cycle. So, it is important to investigate the association of SPEs with sunspot cycle on long-term basis. In this communication, we have find an association of occurrence of SPEs (energy $\geq 10$ $\mathrm{MeV}$ ) and its two kinds (Impulsive and Gradual) with 11-year sunspot cycle, during solar cycle 23, is plotted in Figure 3. These associations haven't shows very significant correlation between the yearly occurrences of SPEs and its two kinds with 11-year sunspot cycle. 


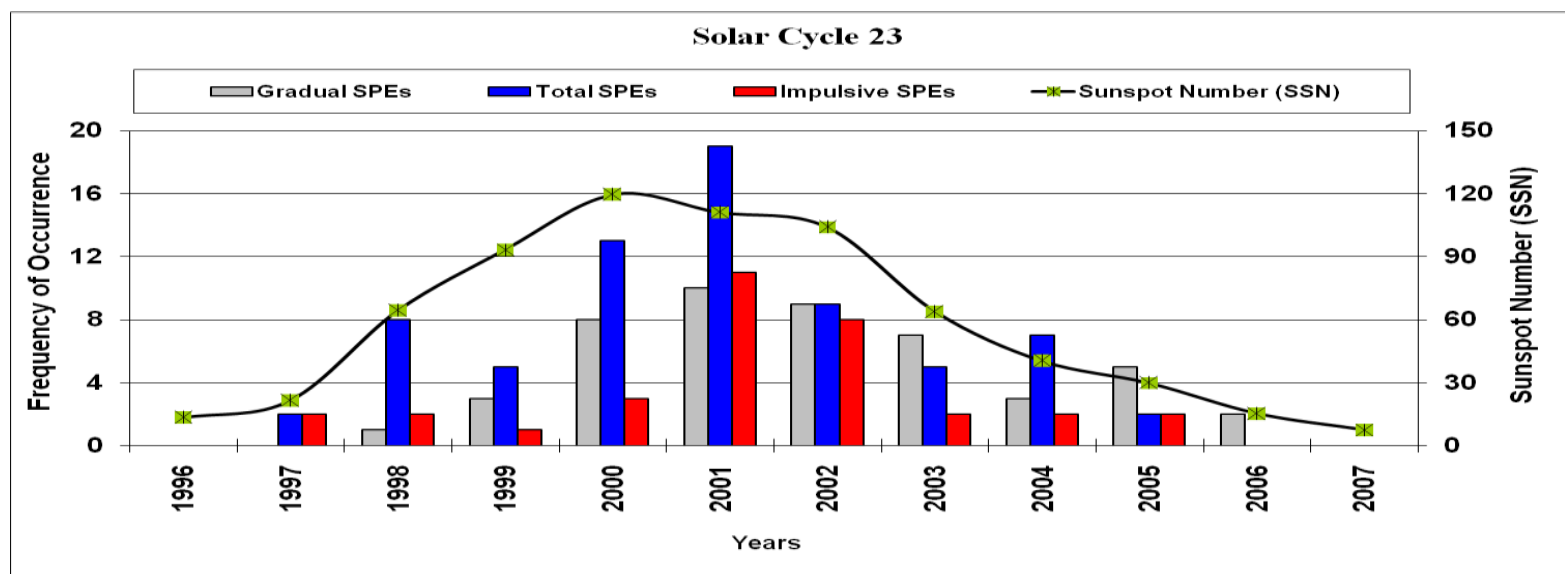

Figure 3: Shows the association of yearly occurrence of solar proton events (SPEs) and their two kinds (Impulsive and Gradual) with 11-year sunspot cycle, observed during the period 19962007.

SPEs (energy $\geq 10 \mathrm{MeV}$ ) produced by processes at the Sun and interplanetary space arrive at Earth and enter the atmosphere over the polar regions, much enhanced ionization is produced at altitudes below $100 \mathrm{~km}$. Ionization at these low altitudes is particularly effective in absorbing HF radio signals and can render HF communications impossible throughout the polar regions. Space Weather Prediction Center (SWPC) monitors the energetic proton detectors on NOAA's GOES satellites to determine when an SPE is in progress. The GOES satellite instrument can determine the presence and temporal profile of an SPE, those observations provide no information about the extent of the area over which those protons enter the atmosphere in the polar region or about which radio propagation paths are affected by the increased ionization. NOAA's Polar Orbiting Environmental Satellites (POES) carry proton detectors similar to GOES. However, each POES satellite transits a polar region twice each orbit and can provide a direct measure of the boundaries and extent of the solar proton fluxes entering the atmosphere during an SPE.

\section{Association Of Large GMEs With Halo CMEs And CIRs}

In the present section, we have shown the association of selected 91 large GMSs with single front-side halo CME (S-type), multiple front-side halo CMEs (M-type) and corotating interaction region (CIR) (C-type). These associations are shown in Figure 4. It is found that 62\% GMSs were caused by single front-side halo CME (S-type), whereas 25\% GMSs were caused by multiple front-side halo CMEs (M-type). Rest 13\% GMSs were caused by corotating interaction region (CIR). We find that there is an east-west asymmetry and northsouth asymmetry as well. We have also shown the association of selected 91 large GMSs with three types of surface source region: active region, quiet region and coronal holes, are shown in Figure 5. We find that $62 \%$ GMSs were caused by active region whereas $10 \%$ GMSs were caused by quiet region. 13\% GMSs were caused by coronal holes and $15 \%$ GMSs are uncertain due to data gap.

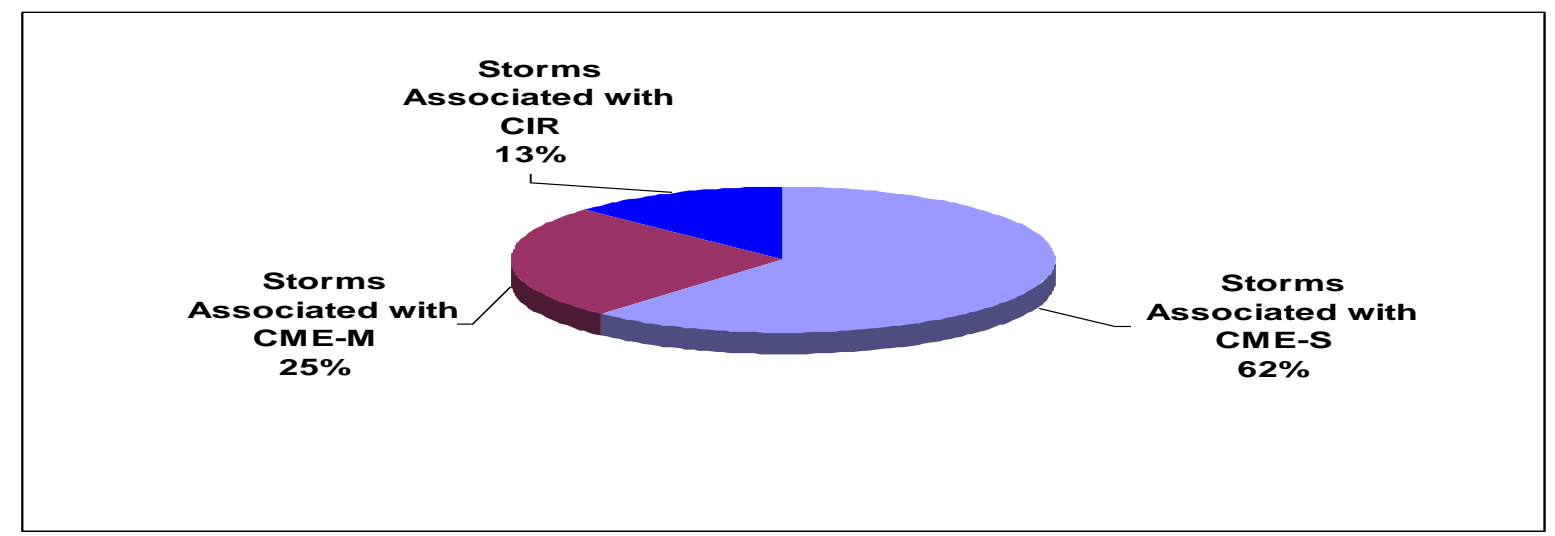

Figure 4: Shows the association of 91 large geomagnetic storms (GMSs) with single front-side halo CME (S-type); multiple front-side halo CMEs (M-type) and corotating interaction region (CIR) (C-type), observed during solar cycle 23. 


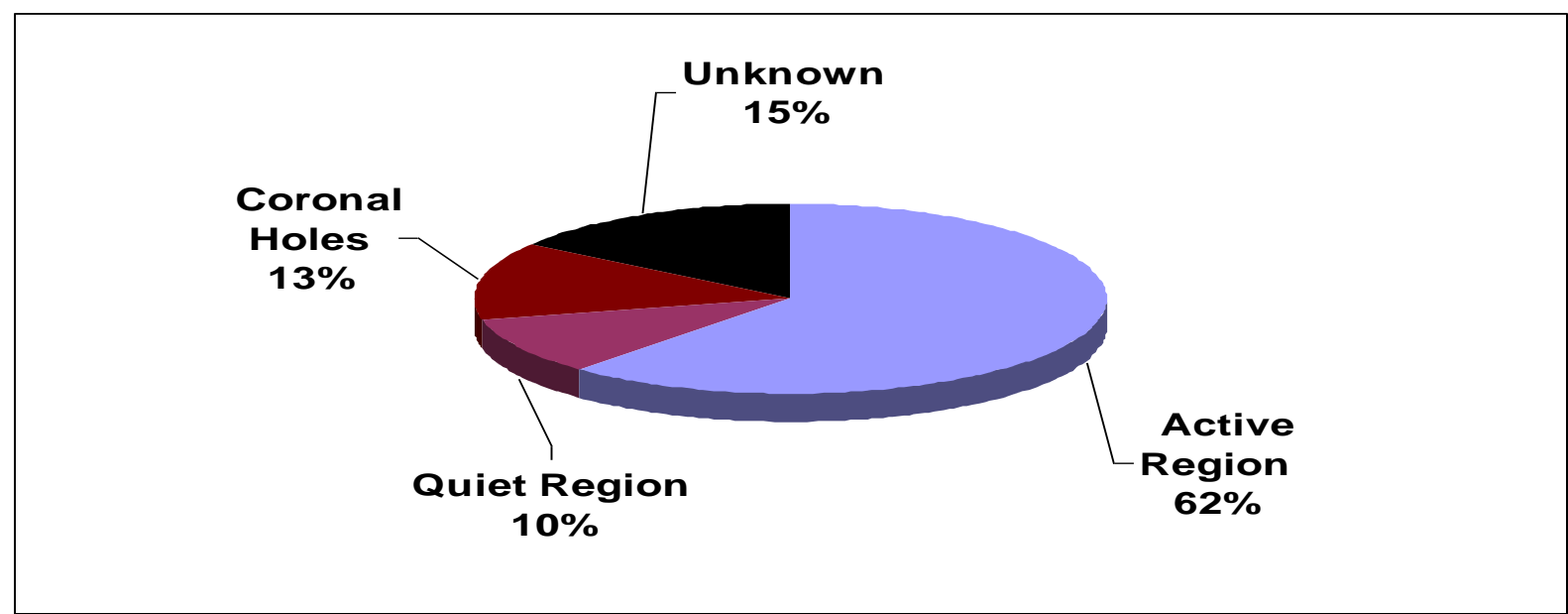

Figure 5: Shows the association of large geomagnetic storms (GMSs) with three types of surface source region: active region, quiet region and coronal holes, observed during solar cycle 23.

\section{Study Of Halo CMEs And Their Geoeffectiveness}

CMEs that appear to surround the occulting disk of the observing coronagraphs in sky-plane projection are known as halo CMEs (Howard et al., 1982). Halo CMEs are fast and wide on the average and are associated with flares of greater X-ray importance because only energetic CMEs expand rapidly to appear above the occulting disk early in the event (Gopalswamy et al., 2007). Extensive observations from LASCO/SOHO have shown that full halos constitute $~ 3.6 \%$ of all CMEs, while CMEs with width $\geq 120^{\circ}$ account for $\sim 11 \%$ (Gopalswamy, 2004). Full halos have an apparent width (W) of $360^{\circ}$, while partial halos have $120^{\circ} \leq \mathrm{W}<360^{\circ}$. Halo CMEs are said to be front-sided if the site of eruption can be identified on the visible disk usually identified as the location of $\mathrm{H}$-alpha flares or filament eruptions. Details on how to identify the solar sources are discussed by Gopalswamy et al (2007). Halos with their sources within $\pm 45^{\circ}$ of the central meridian are known as disk halos, while those with a central meridian distance (CMD) beyond $\pm 45^{\circ}$ but not beyond $\pm 90^{\circ}$ are known as limb halos. Disk halos are likely to arrive at Earth and cause GMSs, while limb halos only impact Earth with their flanks and hence are less geoeffective (Gopalswamy et al., 2007).

In the present investigation, we have analyzed in detail all halo CMEs occurred during solar cycle 23. We have considered here two types of halo CMEs. First, the classical full halo CMEs that appears to surround the entire occulting disk very late, often in the field of view of the $\mathrm{LASCO} / \mathrm{C}_{3}$. Sometimes limb events appear as halo due to deflections of pre-existing coronal structures by the fast CME. Therefore, after very careful examination one can distinguish a real halo CME out of limb fast events deflecting coronal material. We have examined the 354 halo CMEs. Here, all CMEs with angular width only $360^{\circ}$ have been considered as halo CME. In present section, we have analyzed solar cyclic variation of halo CMEs and its different characteristics.

Out of selected 91 large GMSs occurred during solar cycle 23, 56 were associated with halo CMEs. The largest GMS were observed on $20^{\text {th }}$ November 2003. This event can be put in the category of super GMSs. The $\mathrm{D}_{\mathrm{st}}$ magnitude during this event is observed to be $-472 \mathrm{nt}$. This event is associated with halo CME from solar active region. Speed of CME is found to be about $1660 \mathrm{~km} \mathrm{~s}^{-1}$. The histograms of annual distribution of the frequency of halo CME, and their association with annual mean sunspot number is shown in Figure 6. We have found that the occurrence frequency of halo CME generally follows the different phases of solar cycle. During the solar maximum the maximum number of halo CMEs have been occurred. 


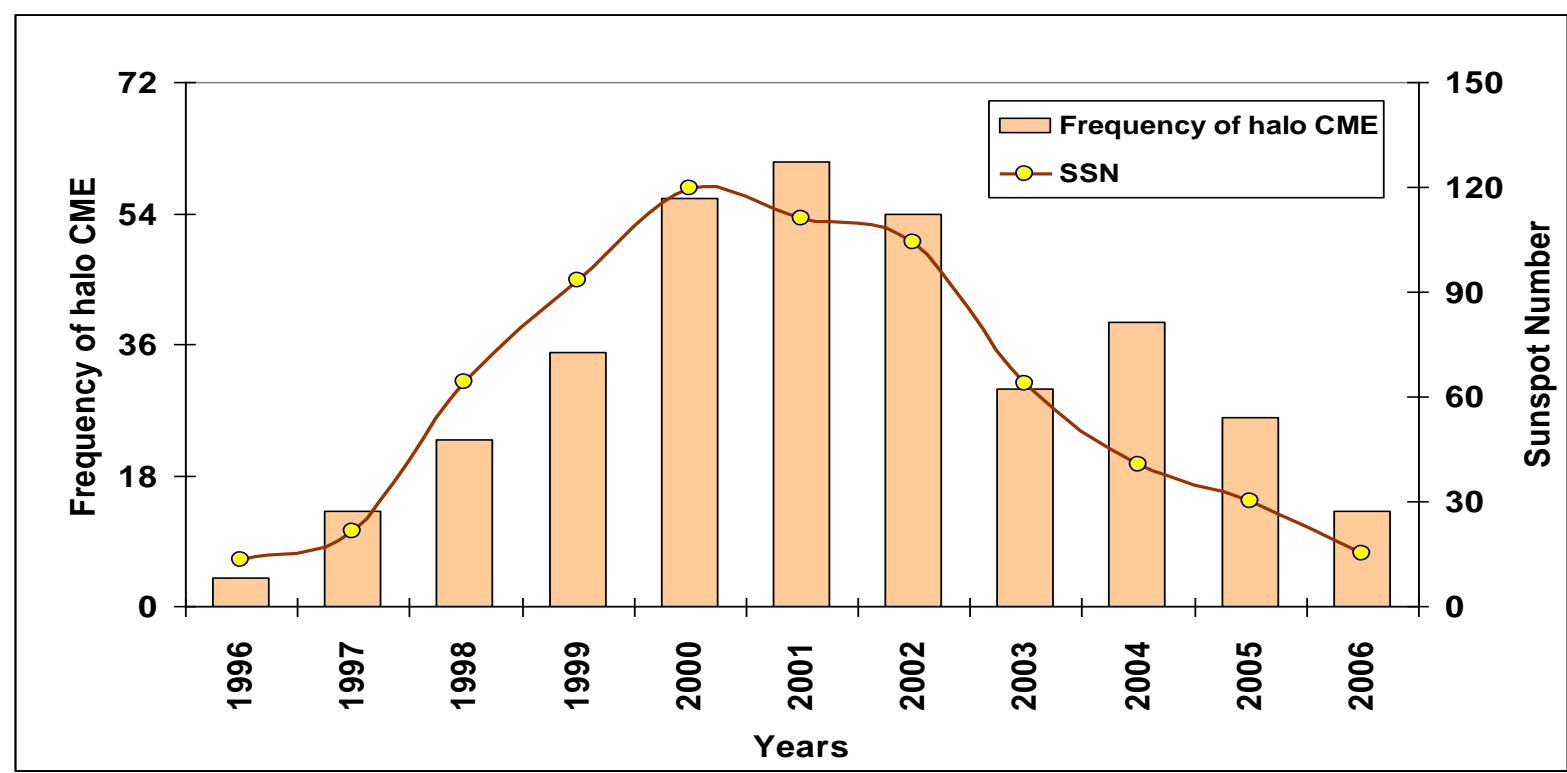

Figure 6: Shows the association of annual frequency of halo CME, and their association with annual mean sunspot number, observed during solar cycle 23.

\section{References}

[1]. Crooker N U, Cliver E W \& Tsurutani B T, J Geophysics Res Lett (USA), 19 (1992) 429.

[2]. Cliver, E.W., Solar Phys., 84 (1983) 347.

[3]. Cane, H.V., McGuire, R. E. and Von Rosenvinge, T. T., Astrophysical J., 301 (1986) 448.

[4]. Gopalswamy, N., The Sun and the Heliosphere as an Integrated System, edited by Poletto, G. and Suess, S. T., Chap. 8 (2004) p 201, Kluwer Acad., Boston.

[5]. Gopalswamy, N., Yashiro, S. and Akiyama, S., J. Geophysics. Res., 112 (2007) A06112.

[6]. Howard, R. A., Michels, D. J., Sheeley, N. R.(Jr.) and Kooman, M. J., ApJ, 263 (1982) L101.

[7]. Forbush, S.E., Phy. Rev., 70 (1946) 771.

[8]. Kahler, S.W., 1992, Annu. Rev. Astron. Astrophys., 30, 113.

[9]. Russell C T \& McPherron R L, Space Sci Rev (Netherlands), 15 (1973) L205. 